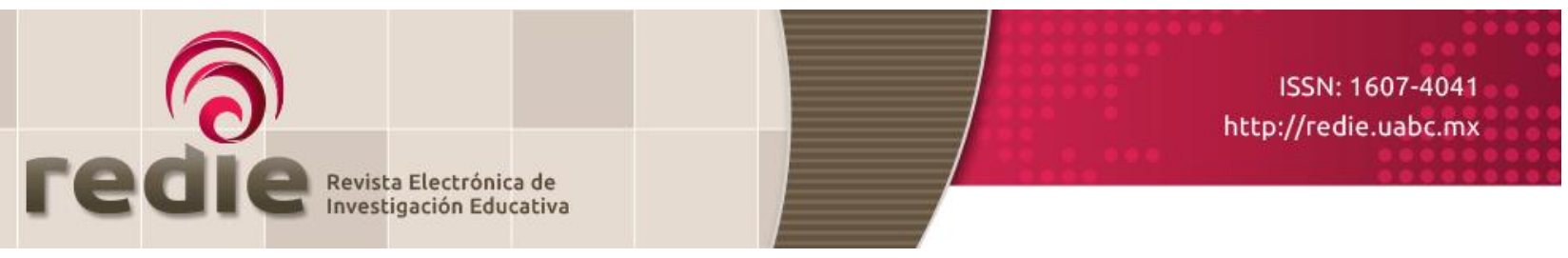

Vol. 22, 2020/e15

\title{
Lectura y escritura epistémicas: movilizando aprendizajes disciplinares en textos escolares
}

\section{Epistemic Reading and Writing: Promoting Disciplinary Learning in Textbooks}

Federico Navarro (1) https://orcid.org/0000-0001-9131-3245

Natalia Ávila Reyes (2) https://orcid.org/0000-0003-3528-6806

Marcelo Cárdenas (2) https://orcid.org/0000-0001-7525-0475

(1) Universidad de O'Higgins

(2) Pontificia Universidad Católica de Chile

(Recibido: 3 de junio de 2018; Aceptado para su publicación: 6 de agosto de 2018)

Cómo citar: Navarro, F., Ávila, N. y Cárdenas, M. (2020). Lectura y escritura epistémicas: movilizando aprendizajes disciplinares en textos escolares. Revista Electrónica de Investigación Educativa, 22, e15, 1-13. https://doi.org/10.24320/redie.2020.22.e15.2493

\section{Resumen}

El texto escolar es objeto de promoción en políticas públicas educativas porque operacionaliza el currículum escolar. Sin embargo, predominan actividades expositivas y reproductivas con escasa atención al potencial epistémico de la lectura y la escritura para promover aprendizajes en las disciplinas. Siguiendo la perspectiva de Escribir para aprender y Aprender a escribir, el propósito de este artículo es presentar una propuesta didáctica fundamentada, sistemática y replicable de enseñanza de la lectura y la escritura en Matemáticas, Ciencias Naturales y Ciencias Sociales de educación básica, materializada en 107 cápsulas didácticas que se incluyeron en 24 libros de texto chilenos entre 2015 y 2017. Se identifica la riqueza y diversidad de géneros discursivos a través del currículum, se seleccionan actividades situadas de libros de texto que incluyan leer o escribir esos géneros, y se construyen secuencias didácticas con socialización de expectativas, modelamiento de proceso y facilitación de recursos y procedimientos lingüísticos.

Palabras clave: Lectura y escritura a través del currículum, enseñanza de la escritura, procesos de escritura, procesos de lectura, tareas de escritura.

\begin{abstract}
Textbooks are central to educational public policies as they operationalize the school curriculum. However, most activities are expositive and reproductive, with little attention to the epistemic potential of reading and writing to promote learning in the disciplines. Drawing from Writing to learn and Learning to write movements, this article aims to depict a theoretically-informed, systematic and replicable pedagogical proposal to teach reading and writing in Mathematics, Natural Sciences and Social Sciences in the basic education curriculum; the proposal was materialized in 107 teaching sequences that were included in 24 Chilean textbooks between 2015 and 2017. The proposal included identifying diverse and engaging genres across the curriculum; selecting textbooks disciplinary activities demanding the reading and writing of those genres; constructing teaching sequences through socializing expectations, modeling processes, and facilitating linguistic resources and procedures.
\end{abstract}


Keywords: Writing across the curriculum, writing instruction, writing processes, reading processes, writing assignments.

\section{Introducción}

En el complejo entramado de recursos educativos que circulan en la escuela, el texto escolar cumple la función de materializar el currículum en actividades didácticas concretas en el aula, mejorar los aprendizajes y ampliar las oportunidades educativas. Esta relevancia educativa del texto escolar ha provocado que su promoción, difusión y distribución forme parte de las políticas públicas de numerosos países latinoamericanos y de otras regiones (Alshwaikh y Morgan, 2014), al punto de que el Banco Mundial afirma que la dotación pública de materiales (como libros de texto) resulta fundamental para garantizar la calidad y equidad educativas (Córdova y Velásquez, 2010).

De esta forma, el texto escolar se considera una herramienta que permitiría llevar al aula propuestas didácticas de avanzada y modelar a los profesores metodologías activas de enseñanza que forman parte del currículum escolar. Sin embargo, esto no siempre ocurre. En el caso de Chile, investigaciones sobre los textos escolares de Ciencias Naturales muestran un bajo desarrollo de la alfabetización científica en sus actividades (Uribe y Ortiz, 2014) y un predominio de la exposición de contenidos sin desarrollo ulterior, en detrimento del desarrollo de habilidades científicas (Meneses et al., 2014). En efecto, pese a que las Bases Curriculares chilenas señalan de manera explícita que el lenguaje es una herramienta de aprendizaje de todas las disciplinas, pocas veces los textos escolares tematizan el uso del lenguaje en las asignaturas (Ministerio de Educación, 2013). La investigación de Meneses et al. (2014) mostró que no sólo no hay un trabajo con el lenguaje académico, sino que la construcción discursiva de los conceptos representaría ante todo un obstáculo para el aprendizaje de las ciencias, ya que la demanda de lecturas de contenido está más allá de lo esperable para el nivel.

Del mismo modo, las Bases Curriculares chilenas ofrecen repertorios de géneros discursivos propios de las disciplinas para estimular su lectura y escritura, como mapas, líneas de tiempo, informes de laboratorio, entre otros, pero éstos no se abordan en el aula en general ni en los textos escolares en particular. Una investigación sobre prácticas de escritura en 6o. año de primaria en Chile mostró que el concepto de escritura para los estudiantes se reduce a la copia de contenidos desde el pizarrón o desde el texto escolar, mientras que los profesores limitan la escritura a redactar respuestas abiertas: la elaboración de géneros escritos se encuentra fuera del repertorio de actividades (Gómez et al., 2016). Un resultado similar exhiben Mateos et al. (2006) en una investigación sobre centros educativos públicos y privados españoles; ellos señalan que, en secundaria, la mayoría de las actividades son de carácter reproductivo, como la copia, la toma de apuntes, el subrayado de lecturas, la extracción de ideas principales o el resumen de un solo texto, mientras que las más infrecuentes las constituyen el comentario, la reflexión o el ensayo y el uso de más de una fuente para construir un texto.

En un estudio etnográfico de caso de tres asignaturas de Ciencias Sociales de una escuela secundaria argentina a la que asisten alumnos de sectores socioeconómicos desfavorecidos, Rosli et al. (2015) también muestran que ni la interpretación de textos ni la escritura sobre lo leído operaron como objetos de enseñanza o instrumentos de aprendizaje; por el contrario, se circunscribieron a actividades de localización y reproducción de información, y la puesta en común fue dominada por la exposición oral sin apoyo textual ni discusión de interpretaciones por parte de los alumnos.

En suma, estudios recientes en diferentes contextos iberoamericanos identifican la necesidad de que los textos escolares y las prácticas docentes fomenten el uso de la lectura y la escritura en las asignaturas como objetos e instrumentos de enseñanza y aprendizaje. Ante esta necesidad, este artículo presenta una propuesta didáctica fundamentada, sistemática y replicable de lectura y escritura epistémicas en las materias de educación básica que se implementó recientemente en el marco del sistema educativo chileno. Entre 2015 y 2017 se relevaron los géneros discursivos que los estudiantes deben leer y escribir según las bases curriculares, se crearon 107 orientaciones o actividades (denominadas cápsulas didácticas), y se incluyeron en 24 libros de texto chilenos de Matemáticas, Ciencias Naturales y Ciencias Sociales. 
Los principios teóricos de esta propuesta se pueden encontrar en los movimientos conocidos como "Escribir para aprender" y "Aprender a escribir" en el marco de la enseñanza de la lectura y la escritura a través del currículum, surgidos en Estados Unidos y otros países de habla inglesa desde fines de la década de los setenta. Estos movimientos experimentaron gradualmente una expansión hacia el aula escolar, alimentados por múltiples influencias desde las ciencias del lenguaje y de la educación. Así, hacia la década del 2000 pasaron a formar parte fundamental de la agenda de innovación educativa, tal como lo atestiguan tres libros clave escritos en español acerca del desarrollo de esta perspectiva: Escribir y leer a través del currículum (Tolchinsky y Simó, 2001) en la primaria; Escribir para aprender: disciplinas y escritura en la escuela secundaria (Navarro y Revel, 2013), para la enseñanza media, y Escribir y comunicarse en contextos científicos y académicos (Castelló, 2007), para la educación superior. La presente propuesta constituye una experiencia concreta de implementación de este modelo innovador en las aulas chilenas de educación primaria a través de la elaboración de materiales escritos en textos escolares destinados a áreas disciplinares.

\section{Fundamentos teóricos}

\subsection{Escribir para aprender}

Con base en una teoría sociocultural del aprendizaje, que entiende el lenguaje como herramienta semiótica que permite internalizar contenidos, Emig (1977) fue una de las pioneras en plantear que la escritura es un modo de procesar e incorporar el conocimiento. La escritura permite disponer los contenidos "fuera" de uno, es decir, funciona como un dispositivo de memoria que permite tomar distancia, observar y manipular el conocimiento. Al respecto, Navarro y Revel (2013) señalan que el conocimiento es construido en el marco de la interacción social, cuya naturaleza es lingüística: se dialoga, se habla, se lee y se escribe para otro, y estos intercambios se dan en un marco sociocultural determinado, que en la escuela corresponde a las disciplinas. En consecuencia, aprender la disciplina no es algo diferente de leerla o escribirla: apropiarse de su lenguaje implica ejercitar la propia disciplina, es decir, sus campos y modos de acción y de construir el conocimiento.

Sin embargo, no cualquier actividad de lectura o escritura promueve el aprendizaje. Como se explicó antes, la mayor parte de las actividades con uso del lenguaje que se realizan en el aula son reproductivas. El potencial epistémico de la escritura (Miras y Solé, 2007) ocurre cuando se propicia una interacción entre la representación de la tarea y dos tipos de espacios: el problema retórico (a quién, cómo y para qué escribir) y el contenido (qué escribir) (Scardamalia y Bereiter, 1992). En otras palabras, el potencial epistémico de la escritura se despliega cuando se fomenta en los estudiantes un interés por aplicar estrategias para resolver una tarea de escritura: planificar, seleccionar contenidos, documentarse, escoger las palabras adecuadas, reformular. De esta manera, los estudiantes usan la escritura como herramienta para transformar el conocimiento: descubren nuevas relaciones entre elementos, formulan críticas, añaden otros conocimientos y experiencias y participan socialmente en las disciplinas por medio de la palabra escrita. El componente epistémico no se restringe a la escritura, también la lectura opera como instrumento para reestructurar y transformar conocimientos y dar forma al pensamiento (Serrano, 2014).

\subsection{Aprender a escribir}

La lectura y la escritura en entornos educativos no sólo constituyen herramientas de aprendizaje, sino que presentan rasgos propios necesarios para comunicarse en los diferentes ámbitos disciplinares que es preciso aprender. Schleppegrell (2004) señala que gran parte del éxito escolar se juega en el desempeño escrito, pero en la práctica la forma de leer y escribir para cada asignatura permanece como una expectativa implícita. Por estos motivos, enseñar de manera explícita a leer y escribir en cada área del conocimiento es útil no sólo para democratizar el acceso al conocimiento y mejorar el rendimiento académico, sino también para ejercitar las diversas formas con las que cada disciplina crea ese conocimiento (Navarro y Revel, 2013).

Meneses et al. (2017) han identificado las características que diferentes autores han definido como usos académicos del lenguaje y las han operacionalizado como conjunto de habilidades claves de lenguaje académico (HCLA) en español con niños chilenos de entre 40. y 6o. grado de enseñanza primaria. Estas habilidades incluyen el empaquetamiento y desempaquetamiento de nominalizaciones, la conexión lógica 
de las ideas, la identificación de anáforas conceptuales, la interpretación de los puntos de vista, la comprensión de términos metalingüísticos, la organización de textos analíticos y la identificación del registro académico; todas ellas con efectos predictores de la comprensión lectora en la escuela (Uccelli y Meneses, 2015). Esta línea de investigación demuestra que entre las estrategias más útiles para desarrollar el lenguaje académico están las instancias de planificación y edición de textos en todas las disciplinas, y que los temas gramaticales deben tratarse al servicio de la construcción de sentido en los textos. Navarro y Revel (2013) señalan también la importancia de revisar, reescribir y editar como estrategia para adquirir las características del lenguaje de las disciplinas.

\subsection{Concepciones sobre escritura}

La comprensión de la lengua escrita como una herramienta de aprendizaje que requiere ser incorporada en todas las disciplinas del currículum escolar se opone a muchas de las concepciones de directivos, profesores y estudiantes, además de editores de textos escolares y otros elaboradores de materiales didácticos, sobre la lectura y, sobre todo, la escritura. Estas concepciones operan muchas veces de forma implícita, es decir, en tanto ideas profundamente arraigadas que cuesta identificar y modificar, pero que se organizan sistemáticamente y median en las prácticas áulicas (Pozo et al., 2006). Algunas de estas concepciones sobre la escritura corresponden a teorías ingenuas o de sentido común, y por lo tanto son socialmente compartidas. Los profesores deben intentar reconocer estas concepciones ingenuas sobre la escritura, con el fin de evitar movilizar acciones arraigadas en su uso como mero método de registro, transcripción, evaluación o reproducción reportadas en investigaciones previas (Mateos et al., 2006). La elaboración de una propuesta didáctica de enseñanza de lectura y escritura epistémica a lo largo del currículum debe fundamentarse en la deconstrucción de las concepciones o mitos sobre la escritura. A continuación se discute una selección de estas concepciones.

La escritura es transcripción. La escritura es una modalidad por sí misma, diferente de la oralidad y del pensamiento. No es oralidad transcrita, porque tiene otras convenciones y características (BlancheBenveniste, 1998): la oralidad suele ser espontánea y en tiempo real, mientras que la escritura implica un medio distinto en el que hay un tiempo diferido para la producción que da espacio a planificar y revisar. Además, la menor dependencia del contexto obliga a representar en el escrito todos los datos de la situación comunicativa necesarios para la comprensión, por lo que se recurre a una organización gramatical distinta a lo oral. Pero la escritura tampoco es una transcripción del pensamiento; por el contrario, es una herramienta para organizar, formular y revisar ideas (Scardamalia y Bereiter, 1992), sin que exista un pensamiento previamente codificado "en la mente" que luego se plasme en el papel. En efecto, el pensamiento se construye al escribir (McCutchen et al., 2008). De esta manera, como la escritura no es una transcripción del pensamiento, no es posible establecer relaciones directas entre la calidad de la escritura de un estudiante y su inteligencia o su conocimiento de un tema. Escribir bien es un proceso complejo y situado que se vincula con el uso de estrategias específicas que permiten a los escritores organizar sus textos con mayor eficiencia y variedad de recursos, pero no se trata de un correlato necesario de lo que el estudiante sabe o puede aprender sobre un contenido disciplinar.

La escritura se aprende al inicio de la escolaridad. Los primeros años de educación básica se focalizan en una serie de destrezas y estrategias relacionadas con dominar el código y producir lenguaje escrito con fluidez. No obstante, la escritura va mucho más allá de la codificación: es una práctica compleja y multidimensional. Cada vez que escribimos un texto nuevo participamos en géneros discursivos que quizá no conocíamos, como cartas de opinión o informes, con sus características, convenciones y expectativas. Del mismo modo, cada ámbito utiliza el lenguaje de una manera diferente, que sólo se aprende cuando participamos de ese nuevo ámbito. Por este motivo el aprendizaje de la escritura se produce en tareas progresivas y en variedad de ámbitos, tanto de educación formal como mediante participación en prácticas vernáculas y profesionales, a lo largo de toda la vida (Bazerman, 2013).

La escritura es talento e inspiración. Pensar la escritura como talento es simplificar su complejidad a una cuestión genética o azarosa, que algunos estudiantes tendrían y otros no, y que puede operar como estigmatización dentro del aula. Si bien es cierto que algunos estudiantes pueden tener mayor facilidad o flexibilidad para adaptarse a situaciones y demandas de escritura, este supuesto talento en realidad revela 
diferentes trayectorias de formación en la escuela, en el ámbito familiar y en otros entornos. Como en la escuela se aprende y evalúa mediante la lengua escrita, ésta debe ser objeto explícito e intencionado de enseñanza, y no puede entenderse como una dotación natural que a algunos estudiantes tocó en suerte y a otros no. De la misma manera, la escritura escolar no puede quedar restringida a una instancia espontánea e individual de inspiración, sin andamiaje ni retroalimentación por parte de los profesores. En realidad se trata de un proceso complejo que integra diferentes etapas que incluyen la planificación de las ideas que se van a escribir y su organización; la textualización o producción concreta mediante los recursos de la lengua y de otros modos con los que se articula (imágenes, colores, formatos); y la revisión del texto escrito. Esta última etapa es particularmente crítica y compleja, y sólo los escritores más formados la consideran un componente fundamental de la escritura. Esta concepción de la escritura como proceso se opone a la idea romántica del escritor "inspirado" que escribe un texto de forma casi automática. Un texto sólido y adecuado esconde un trabajo recursivo de preparación, elaboración de borradores, revisión y maduración hasta alcanzar el producto definitivo.

La escritura es un asunto de lengua. La escritura muchas veces forma parte del llamado "currículum oculto", es decir, del conjunto de habilidades que la escuela presupone y evalúa, pero que no enseña formal y sistemáticamente (Schleppegrell, 2004). Esto se debe muchas veces a la creencia de que la escritura se adquiere de forma natural y no escolarizada, es decir, como la lengua materna. Por el contrario, la escritura es una tecnología relativamente reciente en la historia humana cuyo aprendizaje requiere de una acción escolar sistemática y sostenida en el tiempo (Navarro, 2018). También es frecuente pensar que la escritura es un objeto de enseñanza y aprendizaje exclusivo del área de lengua. Sin embargo, la escritura no es un contenido escolar aislado, sino una actividad situada, con distintos propósitos y características en cada ámbito. En las áreas disciplinares, por ejemplo, existen expectativas diferentes respecto de lo que es escribir correctamente: de qué manera se demuestra una hipótesis, cómo se interactúa con las fuentes expertas, qué rol tiene un gráfico, qué persona gramatical conviene usar, etc. Estas expectativas son en general desconocidas por el profesor de lenguaje, y por tanto la escritura disciplinar debe ser objeto de enseñanza de todas las asignaturas. A su vez, es necesario favorecer las oportunidades de escritura en las áreas disciplinares, ya que facilitan los aprendizajes y su evaluación (Bazerman et al., 2016).

\section{Principios para el diseño de secuencias de aprendizaje de la lectura y la escritura en textos escolares}

La propuesta didáctica presentada en este artículo tuvo lugar entre los años 2015 y 2017, fue liderada por los autores de este artículo y tuvo como objetivo la enseñanza de lectura y escritura epistémicas en las áreas de Matemáticas, Historia y Ciencias Naturales. Para ello se desarrollaron cápsulas didácticas incluidas en la guía del docente de 24 libros de texto que buscaron promover el potencial epistémico de ciertas actividades de todos los manuales de esas áreas. La editorial educativa que publicó los libros de texto cuenta con presencia en más de 20 países y ostenta una posición dominante en Chile; cada libro tuvo un tiraje de 3000 ejemplares, es decir, la propuesta llegó a más de 70000 usuarios.

La elaboración de las cápsulas obedeció al siguiente procedimiento: 1) identificación de géneros discursivos escolares presentes en el currículum de las disciplinas de 10. a 60. grado de educación básica en Chile; 2) capacitación a los editores de textos para que propiciaran la lectura y escritura de dichos géneros en las actividades de los textos; 3 ) identificación de una actividad en la mayoría de las unidades didácticas de cada libro de texto en la que se presentara la oportunidad de reflexionar, leer o escribir alguno de estos géneros (los libros tienen 5 o 6 unidades); y 4) creación de una secuencia didáctica en la que el profesor de la disciplina apoya la lectura o la escritura de los géneros escolares basada en la explicitación de los rasgos lingüísticos y discursivos y el andamiaje de su comprensión y producción. A continuación, se sistematizan, fundamentan y ejemplifican estos pasos.

\subsection{Identificación de géneros en el currículum escolar}

El concepto de género discursivo surge desde los estudios del lenguaje para designar formas prototípicas de la comunicación en diversos ámbitos, con interlocutores, objetivos comunicativos, estructura y rasgos lingüísticos característicos (Bajtín, 2005). En esta investigación se adoptó la concepción contemporánea de 
género discursivo, de base bajtiniana, propuesta por los Estudios Retóricos del Género: los géneros son respuestas discursivas prototípicas a las interacciones sociales, es decir, corresponden a acciones sociales tipificadas en una forma discursiva (Bazerman y Prior, 2005). Esta perspectiva permite la identificación de géneros emergentes vinculados a acciones y prácticas, como "indagación y predicción", junto con otros más convencionalizados en formas lingüísticas reconocibles, como "narrativas de historia personal" o "normas"; al mismo tiempo, habilita comprender la relación entre géneros más puntuales y breves, correspondientes a acciones o actividades específicas, y géneros mayores, correspondientes a actividades más amplias y complejas; por ejemplo, una "explicación de gráficos" aparece dentro de una "comunicación de evidencias".

En el ámbito escolar existen algunos géneros que circulan con mayor frecuencia que en otros espacios: por ejemplo, la "descripción de acciones", "el procedimiento", "la respuesta con justificación", "la narrativa histórica". La tabla I muestra los resultados de un relevo de géneros discursivos presentes en las Bases Curriculares chilenas (Ministerio de Educación, 2013) que guiaron la implementación de estrategias de escritura y lectura para aprender en Ciencias Naturales, Ciencias Sociales y Matemáticas. Para realizar el relevo se analizaron cualitativamente de forma colaborativa entre los autores del artículo las habilidades por área (en algunos casos, secuenciadas) y los Objetivos de Aprendizaje (OA) presentes en las Bases Curriculares chilenas. Este procedimiento, con foco en habilidades, aprendizajes y actividades, permitió la reconstrucción de los géneros discursivos que los estudiantes deben leer, escribir y producir oralmente para aprender, es decir, que pueden utilizarse tanto en su modo oral como escrito y multimodal (por ejemplo, oral con apoyo en gráficos).

Tabla I. Géneros discursivos en las Bases Curriculares chilenas de educación básica

\begin{tabular}{|c|c|}
\hline Área & Géneros discursivos secuenciados \\
\hline $\begin{array}{l}\text { Ciencias } \\
\text { Naturales }\end{array}$ & $\begin{array}{l}\text { Indagación y predicción. } \\
\text { Plan de trabajo. } \\
\text { Registro de observaciones. } \\
\text { Explicación de gráficos. } \\
\text { Comparación de objetos. } \\
\text { Explicaciones a partir de resultados experimentales. } \\
\text { Comunicación de evidencias, reflexiones y conclusiones. }\end{array}$ \\
\hline $\begin{array}{l}\text { Historia, } \\
\text { Geografía y } \\
\text { Ciencias Sociales }\end{array}$ & $\begin{array}{l}\text { Narrativas de historia personal, familiar y comunitaria (Historia). } \\
\text { Narrativa con ubicación de eventos históricos en el tiempo (Historia). } \\
\text { Descripción de costumbres y actividades culturales nacionales (Historia). } \\
\text { Descripción de planos y mapas (Geografía). } \\
\text { Descripción de sistemas naturales, sociales y administrativos (Geografía). } \\
\text { Comparación de aspectos de sociedades para identificar continuidades y cambios. } \\
\text { Comparación de hipótesis y argumentos respecto de un tema. } \\
\text { Opinión basada en fuentes. } \\
\text { Preguntas y respuesta con relación al pasado, al presente o al entorno geográfico. } \\
\text { Comunicación de observaciones y análisis. } \\
\text { Proyectos de acción comunitaria (Formación ciudadana). } \\
\text { Normas (Formación ciudadana). }\end{array}$ \\
\hline Matemáticas & $\begin{array}{l}\text { Explicación de un problema con palabras del estudiante. } \\
\text { Descripción de situaciones del entorno con lenguaje matemático. } \\
\text { Relato basado en una expresión matemática simple. } \\
\text { Descripción verbal de datos. } \\
\text { Descripción de características y posiciones de figuras y representaciones gráficas. } \\
\text { Explicación de relaciones entre números, formas, objetos y conceptos. } \\
\text { Registro de datos en tablas y gráficos. } \\
\text { Explicación de solución de situaciones numéricas. } \\
\text { Preguntas y respuestas formuladas frente a suposiciones y reglas matemáticas. } \\
\text { Problema real creado a partir de una expresión matemática, una ecuación o una representación. } \\
\text { Procedimiento estructurado para resolver problemas. }\end{array}$ \\
\hline
\end{tabular}

Los géneros fueron identificados cualitativamente y se propusieron denominaciones basadas en las etiquetas y descripciones contenidas en las Bases Curriculares. Estos géneros se ordenan según su instancia de aparición a lo largo de los seis años de formación básica. En el caso de Ciencias Naturales, los géneros conforman una secuencia lógica de habilidades vinculadas a la actividad de indagación científica sobre el 
entorno, lo que constituye el objetivo central del área. En el caso de Ciencias Sociales, los géneros se organizan según su nivel de complejidad y la madurez del estudiante, pero también según las especificidades disciplinares dentro del área (aclarado entre paréntesis).

Los resultados muestran una importante diversidad de géneros discursivos, con una secuencia de aparición consistente a lo largo del tiempo. Como se puede observar, se trata -en la mayoría de los casos- de géneros escolares muy breves y con objetivos comunicativos puntuales y situados, y que muchas veces resultan componentes acotados de géneros que realizan actividades sociales mayores.

\subsection{Identificación de actividades en libros de texto}

En esta fase se identificaron actividades existentes en los libros de texto que contuvieran potencial para desarrollar la enseñanza explícita de la lectura y la escritura, o bien, para potenciar el aprendizaje por medio de lo escrito. El procedimiento de identificación tomó en consideración los siguientes criterios: 1) consultar con los editores disciplinares responsables de los libros escolares qué actividades consideraban factibles de intervención; 2) priorizar para la intervención actividades de lectura o escritura complejas, que pudieran incluir pasos para su resolución, por ejemplo colaboración entre pares, planificación y revisión, análisis lingüístico (léxico, gramática); 3) distribuir de forma balanceada a lo largo de los textos escolares las actividades para intervenir (una por unidad), es decir, que no hubiera exceso o ausencia de actividades intervenidas en las distintas unidades. La siguiente figura corresponde a dos actividades de matemáticas para el nivel de 6o. grado:

\section{Consigna 1:}

Francisca le explica a Emiliano cómo restar números enteros. Le dice: "lo que debes hacer es cambiarle el signo al segundo número y iya está!". Emiliano le responde: "entonces cuando tengo 5 - 4 es equivalente a tener $5-(-4)$ ". La equivalencia que presenta Emiliano es falsa. Determina el error de la explicación de Francisca y luego corrígelo.

\section{Consigna 2:}
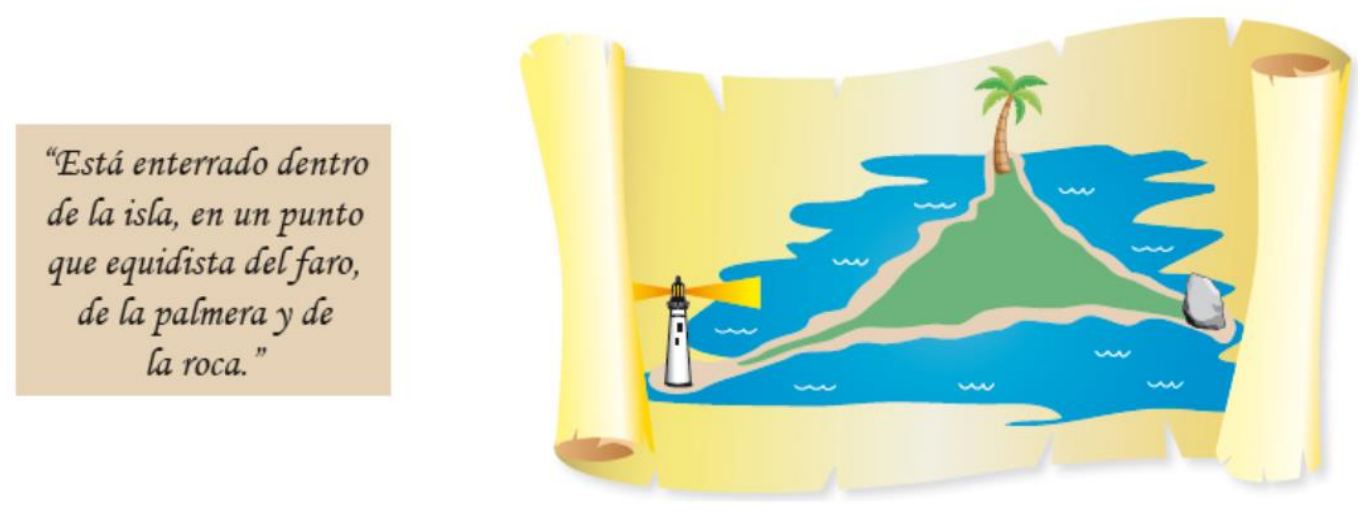

Figura 1. Dos tareas de matemáticas para 6o. grado

En ambos casos las actividades se prestan para trabajar en aula la lectura en Matemáticas. Una débil comprensión de lectura puede muchas veces resultar en un obstáculo para acceder a las explicaciones y mediaciones. Una forma concreta de apoyar este proceso es brindando acceso al vocabulario, lo que permite alivianar la carga cognitiva de la comprensión lectora. El lenguaje utilizado en los textos escolares de Matemáticas contiene muchas veces una fuerte densidad léxica (gran cantidad de palabras de contenido en relación con la cantidad total de palabras escritas), con palabras complejas, de baja frecuencia en la vida 
cotidiana, y muchas de ellas abstractas. En el ejemplo es el caso de palabras como "equivalente" y "equidista". Para mediar adecuadamente la comprensión de estas palabras los docentes necesitan explicitar algunas estrategias de lectura y facilitar el acceso al significado, incluso relacionándolo con los contenidos recientemente vistos en la clase. Aunque estos términos puedan resultar obvios para el profesor disciplinar, la adquisición de las palabras académicas complejas y abstractas es un proceso lento y constituye un gran desafío para los estudiantes, incluso en niveles avanzados de educación.

\subsection{Creación de cápsulas con secuencias didácticas}

La intervención en los textos se basó en modelar el uso que los profesores hacen de la tarea. Esto se ejecuta en el formato de sugerencias detalladas en la guía didáctica sobre cómo implementar pequeñas actividades de clase a partir de la lectura y la escritura disciplinar, ya sea para propiciar los aprendizajes (como en el ejemplo de Matemática) o para enseñar explícitamente los géneros, como el ejemplo de Ciencias Sociales a continuación. Para esto, se siguió un esquema general, transversal a todas las tareas, que incluye tres pasos: socializar expectativas, modelar la producción o la comprensión y facilitar recursos, procedimientos y metalenguaje. Por ejemplo, si el profesor de Ciencias Sociales solicita el género "comparación de hipótesis y argumentos respecto de un tema", debería plantear a sus alumnos los siguientes aspectos (tabla II) para asegurar el éxito de la actividad.

Tabla II. Esquema didáctico transversal para la enseñanza de lectura y escritura a través del currículum

\begin{tabular}{l|l}
\hline Esquema transversal & $\begin{array}{l}\text { Género discursivo: } \\
\text { "Comparación de hipótesis y argumentos respecto de un tema" }\end{array}$ \\
\hline a) Socializar expectativas & $\begin{array}{l}\text { Definir qué es una comparación. } \\
\text { Ejemplificar cómo se escribe una hipótesis y cómo se comparan hipótesis y } \\
\text { argumentos entre sí, por ejemplo a partir de una tabla con fortalezas y } \\
\text { debilidades de cada argumento, consensuada y escrita en conjunto con los } \\
\text { estudiantes en el pizarrón. }\end{array}$ \\
$\begin{array}{l}\text { o la comprensión } \\
\text { Ofrecer estrategias de orden lingüístico y discursivo, como el uso de } \\
\text { conectores de causa y consecuencia al relacionar argumentos o la estrategia } \\
\text { procedimientos recursos, } \\
\text { metalenguaje }\end{array}$ & \begin{tabular}{l} 
de planificar el contenido antes de escribir. \\
\hline
\end{tabular}
\end{tabular}

Con los niveles iniciales, cuando los estudiantes aún producen poco texto escrito, el uso de plantillas mostrando partes y características del texto resulta de utilidad. Con los niveles superiores se puede optar por hacer partícipes y corresponsables a los estudiantes en esta definición y consenso sobre la escritura esperada en el aula.

Estos componentes básicos (ordenados en un esquema general transversal situado en un género discursivo escolar específico) se operacionalizaron en una secuencia didáctica para trabajar las actividades del libro de texto en clase. 


\subsection{Ejemplo de una secuencia en Ciencias Naturales}

A continuación, se presenta el ejemplo de una consigna de ciencias de 20. grado y su secuencia de lectura y escritura de acuerdo con el diseño didáctico propuesto. La figura 2 muestra la consigna presente en el libro de texto que utilizan los estudiantes, mientras que la tabla III detalla la secuencia didáctica propuesta al profesor en la guía del docente:

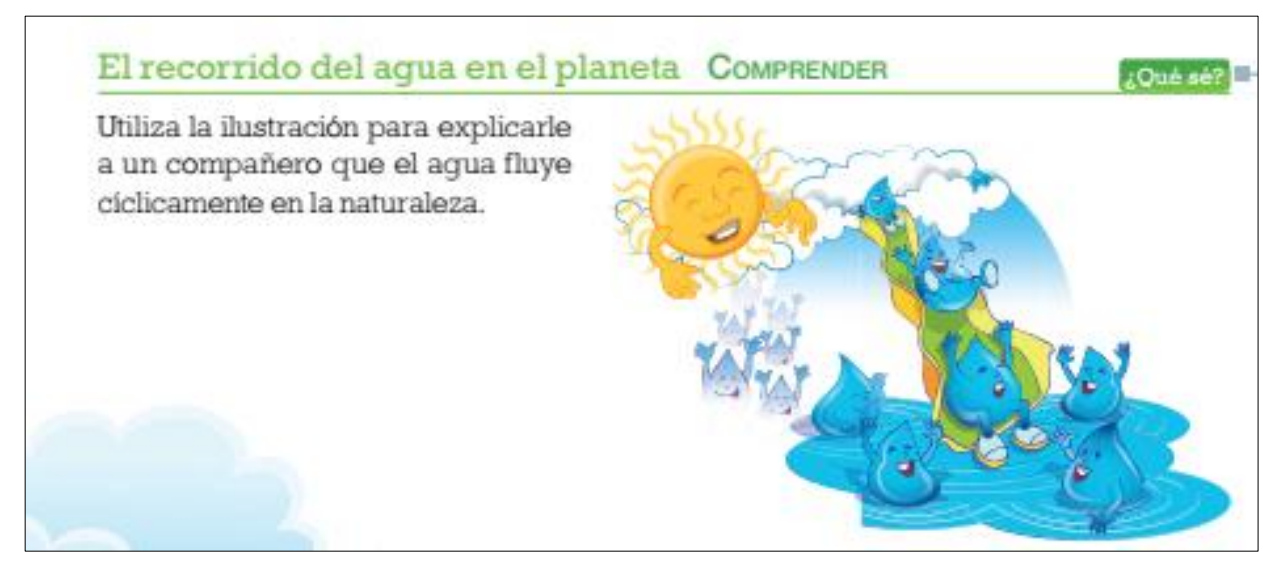

Figura 2. Consigna de Ciencias Naturales de 20. grado en libro de texto para el estudiante 
Tabla III. Secuencia didáctica propuesta al profesor en la guía del docente

\begin{abstract}
Fundamentación
Los estudiantes de 20. básico están en pleno proceso de alfabetización inicial, adquiriendo las habilidades básicas de la escritura escolar. Dentro de esas habilidades, una de las más complejas es la producción de textos que puedan brindar una explicación científica, en ocasiones interactuando con ilustraciones, un rasgo característico del discurso disciplinar de las ciencias.

Este es el caso de la actividad de la página 157. Allí, el niño debe elaborar una explicación verbal oral para una explicación no verbal en forma de ilustración. El contraste entre la explicación verbal y la explicación gráfica muestra algunos rasgos sobresalientes:

1) La explicación gráfica muestra todos los elementos explicativos de forma simultánea.

2) La explicación gráfica no requiere términos técnicos.

3) La explicación gráfica no se articula con una explicación verbal.

Para poder elaborar una explicación oral a partir de la explicación gráfica es preciso que los estudiantes puedan contar con recursos comunicativos para abordar esos contrastes y diferencias. Algunos de esos recursos pueden ser los siguientes:

1) Los elementos simultáneos de la explicación gráfica deben ordenarse mediante organizadores temporales: "primero", "a continuación", "después", "por último".

2) La explicación verbal debe incorporar términos técnicos, en particular ciertos sustantivos para los elementos ("sol", "mar", "océano", "calor", "ciclos") y ciertos verbos para los procesos ("evaporarse", "precipitar",

"absorberse").

3) La explicación verbal requiere vincularse con la explicación gráfica a partir de deícticos y adverbios de locación que indiquen a qué partes de la imagen se vincula: "ahí", "estas gotas", "arriba", "abajo".
\end{abstract}

\title{
Recomendaciones
}

1) Lea la consigna del texto en voz alta. Plantee a los niños el concepto de "explicación". Indague sus conocimientos previos y explicite el objetivo de la actividad: explicar el ciclo del agua.

2) Organice a los niños en equipos para elaborar una planificación de la explicación verbal a partir de la explicación gráfica.

3) Entregue la siguiente tabla y solicite a cada grupo que complete los siguientes elementos. Dé ejemplos en el pizarrón de cómo completar la primera fila de la tabla. Luego supervise el avance en cada equipo. Dé también ejemplos de las palabras técnicas para la explicación (sol, mar, calor, evaporarse, etc.).

\begin{tabular}{l|l|l}
\hline Partes de la explicación & $\begin{array}{l}\text { Palabras para la explicación } \\
\text { (escribir) }\end{array}$ & $\begin{array}{l}\text { Partes de la imagen para cada } \\
\text { parte de la explicación (dibujar) }\end{array}$ \\
\hline Primero, & Sol, calienta & \\
\hline Después, & & \\
\hline A continuación, & & \\
\hline Por último, & & \\
\hline
\end{tabular}

4) Socialice con toda la clase las tablas de cada grupo. Haga sugerencias para revisar y reforzar las planificaciones.

5) Pida a cada grupo que escriba un párrafo con la explicación usando los conectores y las palabras de la tabla.

6) Retroalimente los párrafos y supervise si los textos se comprenden autónomamente sin la necesidad de que el grupo agregue explicaciones orales. Dé tiempo para mejorar las explicaciones antes de cerrar la actividad.

La secuencia didáctica contiene dos componentes generales. En el primero (Fundamentación) se brindan las bases teóricas y pedagógicas que explican el potencial epistémico de la tarea de lectura o escritura. Se hace referencia a los procesos, desafíos y oportunidades de formación en lectura y escritura de los estudiantes de cada nivel educativo. Además, se analizan aspectos específicos de la tarea o actividad. Por último, se brinda a los profesores recursos lingüísticos y discursivos, y un metalenguaje para hablar de ellos. Este primer componente supone que los docentes de las disciplinas no necesariamente cuentan con formación en enseñanza de la lectura y la escritura, a pesar de que puedan reconocer y utilizar el lenguaje disciplinar. Por este motivo, resulta importante proponer elementos teóricos y pedagógicos, con base en una teoría sobre la lengua y sobre la enseñanza de la lectura y la escritura, para habilitar el esquema didáctico general explicado en la sección previa. 
En el segundo componente (Recomendaciones) se ofrece una serie de pasos y actividades concretas para andamiar y modelar la tarea solicitada. Estos pasos y actividades se basan en el esquema didáctico transversal para la enseñanza de lectura y escritura a través del currículum (tabla II): a) explicitar expectativas (por ejemplo, "plantee a los niños el concepto de 'explicación'. Indague sus conocimientos previos y explicite el objetivo de la actividad: explicar el ciclo del agua"); b) modelar la producción o la comprensión (por ejemplo, "pida a cada grupo que escriba un párrafo con la explicación usando los conectores y las palabras de la tabla"); y c) explicitar recursos, procedimientos y metalenguaje ("entregue la siguiente tabla y solicite a cada grupo que complete los siguientes elementos").

\section{Conclusiones}

Esta propuesta didáctica de trabajo con el potencial epistémico de la lectura y la escritura en las disciplinas escolares (Castelló, 2007; Navarro y Revel, 2013; Tolchinsky y Simó, 2001) ofrece un modelo teóricamente fundamentado, sencillo, replicable y factible para escribir y leer para aprender conocimientos disciplinares y para aprender a leer y escribir lenguaje académico. Su inscripción en libros de texto a través del currículum es un camino estratégico para llegar al aula mediante un instrumento con orientación, andamiaje y retroalimentación que, según numerosas investigaciones, mejora los aprendizajes y reduce la inequidad educativa (Córdova y Velásquez, 2010). El estudio de las Bases Curriculares de educación básica chilenas (Ministerio de Educación, 2013) ha revelado que, lejos de ser un añadido o un obstáculo a los contenidos disciplinares (Meneses et al., 2014), existe una enorme riqueza, diversidad y consistencia de acciones y actividades sociales tipificadas vinculadas a la lectura y la escritura que pueden ser aprovechadas por los profesores y las profesoras, por los funcionarios de instituciones y organizaciones educativas y por los editores a cargo de textos escolares y guías del docente.

La secuencia didáctica propuesta incluye tres pasos: socializar expectativas; modelar la producción o la comprensión; y facilitar recursos, procedimientos y metalenguaje. Al mostrar ejemplos o modelar en el pizarrón escribiendo una respuesta propia, se explicita qué constituye para el profesor un escrito adecuado. Explicar el proceso de pensamiento y toma de decisiones brinda a los estudiantes modelos de razonamiento que pueden incorporar. Es importante referirse explícitamente a la forma de la escritura y a los recursos de lenguaje usados (conectores, ordenadores, sustantivos, palabras especializadas, etc.). Al modelar la tarea de escritura se brinda a los estudiantes repertorios de estrategias en las que se pueden basar al comenzar cada nueva tarea. Por último, proporcionar tiempo, guías, pautas y ejemplos de cómo revisar y editar fomenta que los estudiantes incorporen de forma gradual estrategias de escritura como proceso. En suma, la secuencia didáctica propuesta y ejemplificada en este artículo permite que se promuevan, amplíen y profundicen tareas de comunicación escrita situadas, explícitas, significativas y orientadas a la transformación del conocimiento, que evitan la mera reproducción de saberes, una práctica pedagógica extendida (Mateos et al., 2006).

Dado que los libros de texto innovados con esta propuesta didáctica tienen actualmente un amplio uso en Chile, investigaciones futuras deberían abordar las formas de implementación en aula de las actividades propuestas, el impacto en los aprendizajes y en los desempeños, y las percepciones de funcionarios, profesores y estudiantes.

\section{Agradecimientos}

Se agradece el financiamiento otorgado por ANID/PIA/Fondos Basales para Centros de Excelencia FB0003, por el Proyecto FONDECYT No 1191069 de ANID y por el Plan de Mejoramiento Institucional (PMI) UCH1501 del Ministerio de Educación, Chile.

\section{Referencias}

Alshwaikh, J. y Morgan, C. (2014). The creation of mathematics in school textbooks: Palestine and England as examples. En K. Jones, C. Bokhove, G. Howson y L. Fan (Eds.), Proceedings of the International Conference on Mathematics Textbook Research and Development (pp. 141-146). The University of Southampton.

https://eprints.soton.ac.uk/374809/ 
Bajtín, M. M. (2005). Estética de la creación verbal. Siglo XXI.

Bazerman, C. (2013). Understanding the lifelong journey of writing development. Journal for the Study of Education and Development. Infancia y Aprendizaje, 36(4), 421-441.

https://doi.org/10.1174/021037013808200320

Bazerman, C. y Prior, P. (2005). Participating in emergent socio-literate worlds: genre, disciplinarity, interdisciplinarity. En R. Beach (Ed.), Multidisciplinary perspectives on literacy research (pp. 133-178).

Hampton Press.

Bazerman, C., Little, J., Bethel, L., Chavkin, T., Fouquette, D. y Garufis, J. (2016). Escribir a través del currículum. Una guía de referencia (F. Navarro, Ed.). Universidad Nacional de Córdoba.

https://rdu.unc.edu.ar/handle/11086/4030

Blanche-Benveniste, C. (1998). Estudios linguísticos sobre la relación entre oralidad y escritura. Gedisa.

Castelló, M. (Ed.). (2007). Escribir y comunicarse en contextos científicos y académicos. Graó.

Córdova, A. y Velásquez, M. (2010). Representaciones sociales acerca de los textos escolares de lenguaje y comunicación de un grupo de estudiantes en proceso de práctica docente final. En Seminario internacional: Textos escolares de lenguaje y comunicación (pp. 272-283). Ministerio de Educación de Chile.

https://issuu.com/paulinainzunza/docs/libro sem tx lenguaje vf $1101 \quad 11 \mathrm{p}$

Emig, J. (1977). Writing as a mode of learning. College Composition and Communication, 28(2), 122-128. https://doi.org/10.2307/356095

Gómez, G., Sotomayor, C., Jéldrez, E., Bedwell, P., Domínguez, A. M., Ávila, N. y Calderón, M. (2016). La producción escrita de estudiantes y escuela en base a resultados SIMCE, factores contextuales y modelos de buenas prácticas docentes. Informe final proyecto FONIDE No. 911437 FONIDE-Mineduc.

https://centroestudios.mineduc.cl/wp-content/uploads/sites/100/2017/07/INFORME-FINAL-F911437.pdf

Mateos, M., Martín, E. y Villalón, R. (2006). La percepción de profesores y alumnos en la educación secundaria sobre las tareas de lectura y escritura que realizan para aprender. En J. I. Pozo, N. Scheuer, M. del Puy, M. Mateos, E. Martín y M. de la Cruz (Eds.), Nuevas formas de pensar la enseñanza y el aprendizaje (pp. 231-242). Graó.

McCutchen, D., Teske, P. y Bankston, C. (2008). Writing and cognition: implications of the cognitive architecture for learning to write and writing to learn. En C. Bazerman (Ed.), Handbook of research on writing: history, society, school, individual, text (pp. 451-470). Taylor \& Francis.

Meneses, A., Montenegro, M. y Ruiz, M. (2013). Calidad de textos escolares para aprender ciencias: habilidades, contenidos y lenguaje académico. En M. de la Cerda (Ed.), Evidencias para políticas públicas en educación: selección de investigaciones, sexto concurso FONIDE (pp. 233-277). Ministerio de Educación de Chile.

Meneses, A., Uccelli, P., Santelices, M. V., Ruiz, M., Acevedo, D. y Figueroa, J. (2017). Academic language as a predictor of reading comprehension in monolingual spanish-speaking readers: evidence from chilean early adolescents. Reading Research Quarterly, 53(2), 223-247. https://doi.org/10.1002/rrq.192

Ministerio de Educación de Chile (2013). Bases Curriculares de la Educación Básica.

http://www.curriculumenlineamineduc.cl/605/articles-30013_recurso_14.pdf (enlace inactivo)

Miras, M. y Solé, I. (2007). La elaboración del conocimiento científico y académico. En M. Castelló (Ed.), Escribir y comunicarse en contextos científicos y académicos: conocimientos y estrategias (pp. 83-112). Graó. 
Navarro, F. (2018). Más allá de la alfabetización académica: cinco funciones de la escritura en educación superior. En M. A. Alves y V. Lensen (Eds.), Formação de Professores: Ensino, linguagens e tecnologias [Formación del profesorado: enseñanza, idiomas y tecnología] (pp. 13-49). Editora Fi.

Navarro, F. y Revel, A. (2013). Escribir para aprender. Disciplinas y escritura en la escuela secundaria. Paidós.

Pozo, J. I., Scheuer, N., Mateos, M. y del Puy, M. (2006). Las teorías implícitas sobre el aprendizaje y la enseñanza. En J. I. Pozo, N. Scheuer, M. del Puy, M. Mateos, E. Martín y M. de la Cruz (Eds.), Nuevas formas de pensar la enseñanza y el aprendizaje: las concepciones de profesores y alumnos (pp. 95-134). Graó.

Rosli, N., Carlino, P. y Roni, C. (2015). Retención escolar y educación de calidad: logros y desafíos pendientes en una escuela secundaria argentina. Archivos Analíticos de Políticas Educativas, 23(102), 1-32.

https://doi.org/10.14507/epaa.v23.1911

Scardamalia, M. y Bereiter, C. (1992). Dos modelos explicativos de los procesos de composición escrita. Journal for the Study of Education and Development. Infancia y Aprendizaje, 58, 43-64.

https://doi.org/10.1080/02103702.1992.10822332

Schleppegrell, M. J. (2004). The language of schooling: a functional linguistics perspective. Lawrence Erlbaum Associates.

Serrano, S. (2014). La lectura, la escritura y el pensamiento. Función epistémica e implicaciones pedagógicas. Lenguaje, 42(1), 97-122. https://doi.org/10.25100/lenquaje.v42i1.4980

Tolchinsky, L. y Simó, R. (2001). Escribir y leer a través del currículum. Horsori.

Uccelli, P. y Meneses, A. (2015). Habilidades de lenguaje académico y su aplicación con la comprensión de la lectura en la escuela primaria y media: un nuevo constructo operacional. Miríada Hispánica, 10, 179-206.

Uribe, M. E. y Ortiz, I. (2014). Programas de estudio y textos escolares para la enseñanza secundaria en Chile: ¿qué oportunidades de alfabetización científica ofrecen? Enseñanza de las Ciencias, 32(3), 37-52. https://doi.org/10.5565/rev/ensciencias.968 